\title{
ON THE STRUCTURE OF IDEMPOTENT SEMIGROUPS
}

\author{
R. J. WARNE
}

\begin{abstract}
An idempotent semigroup (band) is a semigroup in which every element is an idempotent. We describe the structure of idempotent semigroups in terms of semilattices $\Omega$, partial chains $\Omega$ of left zero semigroups, and partial chains $\Omega$ of right zero semigroups. We also describe bands of maximal left zero semigroups in terms of partial chains $\Omega$ of left zero semigroups and semilattices $\Omega$ of right zero semigroups.
\end{abstract}

An idempotent semigroup (band) is a semigroup in which every element is an idempotent. We describe the structure of idempotent semigroups in terms of semilattices $\Omega$, partial chains $\Omega$ of left zero semigroups, and partial chains $\Omega$ of right zero semigroups. We also describe bands of maximal left zero semigroups in terms of partial chains $\Omega$ of left zero semigroups and semilattices $\Omega$ of right zero semigroups.

Unless otherwise specified we employ the definitions and notation of [2]. The following theorem is a starting point in the proof of both of our structure theorems.

TheOREM 1 (Clifford [1], MCLeAN [3]). Let $E$ be an idempotent semigroup. Then, $E$ is a semilattice $\Omega$ of rectangular bands $\left(E_{\delta}: \delta \in \Omega\right)$.

We begin by introducing the following concepts.

Let $W$ be a partial groupoid which is a union of a collection of pairwise disjoint subsemigroups $\left(T_{\delta}: \delta \in \Lambda\right)$ where $\Lambda$ is a semilattice. If $x \in T_{v}$, $y \in T_{\delta}$, and $\delta \leqq \nu$ (in $\Lambda$ ) imply $x y$ is defined (in $W$ ) and $x y \in T_{\delta}$ and if $\xi \leqq \delta$ and $z \in T_{\xi}$ imply $(x y) z=x(y z), W$ is termed a (lower) partial chain $\Lambda$ of the semigroups $\left(T_{\delta}: \delta \in \Lambda\right)$. If $x \in T_{v}, y \in T_{\delta}$, and $\nu \leqq \delta$ imply $x y$ is defined (in $W$ ) and $x y \in T_{v}$, and $\xi \geqq \delta$ and $z \in T_{\xi}$ imply $(x y) z=x(y z), W$ is termed an (upper) partial chain of the semigroups $\left(T_{\delta}: \delta \in \Lambda\right)$.

Received by the editors August 9, 1971.

AMS (MOS) subject classifications (1970). Primary $20 \mathrm{M} 10$.

Key words and phrases. Idempotent semigroup, band of left zero semigroups, band, semilattice, semilattice of right zero semigroups. 
We are now in a position to give the first theorem.

Let $\Omega$ be a semilattice, let $I$ be a (lower) partial chain $\Omega$ of left zero semigroups $\left(I_{\delta}: \delta \in \Lambda\right)$, and let $J$ be an (upper) partial chain $\Omega$ of right zero semigroups $\left(J_{\delta}: \delta \in \Lambda\right)$. Let $\alpha$ be a mapping of $J \times I$ into $I$ and let $\beta$ be a mapping of $J \times I$ into $J$ subject to the conditions:

I. If $r, s \in \Omega,\left(J_{r} \times I_{s}\right) \alpha \subseteq I_{r s}$ and $\left(J_{r} \times I_{s}\right) \beta \subseteq J_{r s}$.

II. If $j \in J_{s}, p \in I_{t}, q \in J_{t}$, and $m \in I_{g}$,

$$
\begin{aligned}
(j, p) \alpha((j, p) \beta q, m) \alpha & =(j, p((q, m) \alpha)) \alpha \quad \text { and } \\
(j, p((q, m) \alpha)) \beta(q, m) \beta & =((j, p) \beta q, m) \beta .
\end{aligned}
$$

Let $(\Omega, I, J, \alpha, \beta)$ denote $\bigcup\left(I_{s} \times J_{s}: s \in \Omega\right)$ under the multiplication $(i, j)(p, q)=(i((j, p) \alpha),(j, p) \beta q)$.

THEOREM 2. ${ }^{1} \quad E$ is an idempotent semigroup if and only if $E \cong(\Omega, I, J, \alpha, \beta)$ for some collection $\Omega, I, J, \alpha, \beta$.

Proof. Let $E$ be an idempotent semigroup. Select and fix an $\mathscr{L}$ class $I_{\delta}$ of $E_{\delta}$ and select and fix an $\mathscr{R}$-class $J_{\delta}$ of $E_{\delta}(\mathscr{L}$ and $\mathscr{R}$ are Green's relations [2]). Thus every element of $E$ may be expressed uniquely in the form $x=i j$ where $i \in I_{\delta}$ and $j \in J_{\delta}$ for some $\delta \in \Omega$. If $e \in I_{\delta}, f \in I_{v}$ and $\nu \leqq \delta,(e f, f) \in \mathscr{L}\left(\in E_{v}\right)$ and, hence, ef $\in I_{v}$. Let $I=\bigcup\left(I_{\delta}: \delta \in \Omega\right)$ and, if $a, b \in I$, define $a \circ b=a b$ (product in $E$ ) if $a b \in I$ while $a \circ b$ is undefined if $a b \bar{\in} I$. Hence, the partial groupoid $(I, \circ)$ is a (lower) partial chain $\Omega$ of left zero semigroups $\left(I_{\delta}: \delta \in \Omega\right.$ ) (since no confusion will arise, we replace "o" by juxtaposition). Similarly, $J=\bigcup\left(J_{\delta}: \delta \in \Omega\right)$ is an (upper) partial chain $\Omega$ of right zero semigroups $\left(J_{\delta}: \delta \in \Omega\right)$. We may define a mapping $\alpha$ of $J \times I$ into $I$ and a mapping $\beta$ of $J \times I$ into $J$ satisfying $I$ by the expression $j i=(j, i) \alpha(j, i) \beta$ where $j \in J_{r}$ and $i \in I_{s}$, say. If $j \in J_{s}, p \in I_{t}, q \in J_{t}$, and $m \in I_{g}$,

$$
((j p) q) m=(j, p) \alpha((j, p) \beta q m)=(j, p) \alpha((j, p) \beta q, m) \alpha((j, p) \beta q, m) \beta
$$

and

$$
j(p(q m))=j(p((q, m) \alpha(q, m) \beta))=(j p((q, m) \alpha))
$$

\footnotetext{
1 The referee informs me that a result similar to Theorem 2 has also been obtained by Petrich (unpublished).
} 
$(q, m) \beta=(j, p((q, m) \alpha)) \alpha(j, p((q, m) \alpha)) \beta(q, m) \beta$ and II follows. Since

$$
(i j)(p q)=i(j p) q=(i((j, p) \alpha))((j, p) \beta q)
$$

for $i \in I_{s}, j \in J_{s}, p \in I_{t}$, and $q \in J_{t}$, say, $(i j) \varphi=(i, j)$ defines an isomorphism of $S$ onto $(\Omega, I, J, \alpha, \beta)$. We next show that $T=(\Omega, I, J, \alpha, \beta)$ is a band. We utilize I to establish closure and II to establish associativity while $(i, j) \in I_{s} \times J_{s}$ implies $(i, j)^{2}=(i, j)$ by a routine calculation.

We will need the following definition.

A partial transformation $\lambda$ of a partial groupoid $W$ is termed an inner left translation of $W$ determined by $e \in W$ if the domain $D$ of $\lambda$ is the set of $s \in W$ such that $e s$ is defined and $s \lambda=e s, \forall s \in D$. We write $\lambda=\lambda_{e}$.

We next give a structure theorem for bands of maximal left zero semigroups.

Let $X$ be a semilattice $\Omega$ of right zero semigroups $\left(X_{\delta}: \delta \in \Omega\right)$. For each $\delta \in \Omega$, select $e_{\delta} \in X_{\delta}$ and let $B=\left(e_{\delta}: \delta \in \Omega\right)$. Under the order, $e_{\beta} \leqq e_{\delta}$ if $e_{\delta} e_{\beta}=e_{\beta}, B$ is a semilattice order isomorphic to $\Omega$. Let $W$ be a (lower) partial chain $B$ of left zero semigroups $\left(T_{e_{\delta}}: e_{\delta} \in B\right)$. For each $s \in X_{\delta}$, let $s^{\prime}=e_{\delta}$. Let $r \rightarrow \alpha_{r}$ be a mapping of $X$ into $\mathscr{T}_{W}$, the full transformation semigroup on $W$, subject to the conditions:

I(a) $T_{e_{\delta}} \alpha_{r} \subseteq T_{\left(r_{\delta}\right)^{\prime}}$;

(b) $\left(g_{e_{\delta}} h_{e_{\beta}}\right) \alpha_{r}=\left(g_{e_{\delta}} \alpha_{r}\right)\left(h_{e_{\beta}} \alpha_{r e_{\delta}}\right)$ for $g_{e_{\delta}} \in T_{e_{\delta}}, h_{e_{\beta}} \in T_{e_{\beta}}$, and $e_{\beta} \leqq e_{\delta}$.

II. $\alpha_{s t} \lambda_{e}=\alpha_{t} \alpha_{s}$ for all $e \in T_{(s t)}$, where $\lambda_{e}$ is the inner left translation of $W$ determined by $e$.

Let $(X, W, \alpha)$ denote $\left\{\left(g_{s^{\prime}}, s\right): s \in X, g_{s^{\prime}} \in T_{s^{\prime}}\right\}$ under the multiplication $\left(g_{s^{\prime}}, s\right)\left(h_{t^{\prime}}, t\right)=\left(g_{s^{\prime}}\left(h_{t^{\prime}} \alpha_{s}\right), s t\right)$.

THEOREM 3. E is a band of maximal left zero semigroups if and only if $E \cong(X, W, \alpha)$ for some collection $X, W, \alpha$.

Proof. Let $E$ be a band $b$ of maximal left zero semigroups. Hence, $b=\mathscr{L}$ and $E / \mathscr{L}=X$ is a semilattice $\Omega$ of right zero semigroups $\left(X_{\delta}: \delta \in \Omega\right)$ where $X_{\delta}=E_{\delta} \mathscr{L}$. If $T_{s}=s \mathscr{L}^{-1}(s \in X),\left(T_{s}: s \in X\right)$ is the collection of $\mathscr{L}$ classes of $E$ with $T_{s} T_{t} \subseteq T_{s t}$. Let $u_{s}$ be a representative element for $T_{s}$. For each $\delta \in \Omega$, select $e_{\delta} \in X_{\delta}$, and, if $s \in X_{\delta}$, let $s^{\prime}=e_{\delta}$. Hence, every element of $E$ may be uniquely expressed in the form $x=g_{s^{\prime}} u_{s}$ where $g_{s^{\prime}} \in T_{s^{\prime}}$. If we let $B=\left(e_{\delta}: \delta \in \Omega\right)$, then, under the order $e_{\beta} \leqq e_{\delta}$ if $e_{\delta} e_{\beta}=e_{\beta}, B$ is a semilattice order isomorphic to $\Omega$. As above, $W=\bigcup\left(T_{e_{\delta}}: e_{\delta} \in B\right)$ is a (lower) partial chain $B$ of left zero semigroups $\left(T_{e_{\delta}}: e_{\delta} \in B\right)$. For each $r \in X$, the expression $u_{r} g_{e_{\delta}}=\left(g_{e_{\delta}} \alpha_{r}\right) u_{r e_{\delta}}$ defines a unique $\alpha_{r} \in \mathscr{T}_{W}$ satisfying I(a). We 
obtain I(b) from the expression

$$
\begin{aligned}
\left(g_{e_{\delta}} h_{e_{\beta}}\right) \alpha_{r} u_{r e_{\delta} e_{\beta}} & =u_{r}\left(g_{e_{\delta}} h_{e_{\beta}}\right)=\left(u_{r} g_{e_{\delta}}\right) h_{e_{\beta}} \\
& =\left(g_{e_{\delta}} \alpha_{r}\right)\left(u_{r e_{\delta}} h_{e_{\beta}}\right)=\left(g_{e_{\delta}} \alpha_{r}\right)\left(h_{e_{\beta}} \alpha_{r e_{\delta}}\right) u_{r e_{\delta} e_{\beta}}
\end{aligned}
$$

where $e_{\beta} \leqq e_{\delta}$. We may write $u_{s} u_{t}=f_{s, t} u_{s t}$ where $f_{s, t} \in T_{(s t)}$. Hence, we obtain II from the expression

$$
\begin{aligned}
f_{s, t}\left(g_{z^{\prime}} \alpha_{s t}\right) u_{s t z^{\prime}} & =f_{s, t}\left(u_{s t} g_{z^{\prime}}\right)=\left(f_{s, t} u_{s t}\right) g_{z^{\prime}}=u_{s}\left(u_{t} g_{z^{\prime}}\right)=u_{s}\left(g_{z^{\prime}} \alpha_{t} u_{t z^{\prime}}\right) \\
& =g_{z^{\prime}} \alpha_{t} \alpha_{s} u_{s\left(t z^{\prime}\right)} u_{t z^{\prime}}=g_{z^{\prime}} \alpha_{t} \alpha_{s} f_{s\left(t z^{\prime}\right)^{\prime}, t z^{\prime}} u_{s t z^{\prime}}=g_{z^{\prime}} \alpha_{t} \alpha_{s} u_{s t z^{\prime}}
\end{aligned}
$$

The last equality follows since $g_{z^{\prime}} \alpha_{t} \alpha_{s}$ and $f_{s\left(t z^{\prime}\right)^{\prime}, t z^{\prime}}$ are both contained in the same $\mathscr{L}$-class of $E$. We have

$$
\begin{aligned}
\left(g_{s^{\prime}} u_{s}\right)\left(h_{t^{\prime}} u_{t}\right) & =g_{s^{\prime}}\left(u_{s^{\prime}} h_{t^{\prime}}\right) u_{t}=g_{s^{\prime}}\left(h_{t^{\prime}} \alpha_{s}\right) u_{s t^{\prime}} u_{t}=g_{s^{\prime}}\left(h_{t^{\prime}} \alpha_{s}\right) f_{s t^{\prime} \cdot t^{\prime}} u_{s t} \\
& =g_{s^{\prime}}\left(h_{t^{\prime}} \alpha_{s}\right) u_{s t}
\end{aligned}
$$

The last equality follows since $h_{t^{\prime}} \alpha_{s}$ and $f_{s t^{\prime}, t}$ are contained in the same $\mathscr{L}$-class of $E$. Hence, $\left(g_{s^{\prime}} u_{s}\right) \varphi=\left(g_{s^{\prime}}, s\right)$ defines an isomorphism of $S$ onto $(X, W, \alpha)$. Next, we show that $(X, W, \alpha)$ is a band of maximal left zero semigroups. We utilize $I(a)$ to establish a closure and $I(b)$ and II to establish associativity. If we let $L_{s}=\left(\left(g_{s^{\prime}}, s\right): g_{s^{\prime}} \in T_{s^{\prime}}\right), E$ is the band $X$ of maximal left zero semigroups $\left(L_{s}: s \in X\right)$.

REMARK. Using the previous proof, $E$ is a band of maximal left zero semigroups if and only if $E$ is a band and $\mathscr{L}$ is a congruence on $E$.

REMARK (ADDED IN PROOF). We may show that "left zero semigroups" may be replaced by "left groups" in Theorem 3 provided we make the following modifications: In the definition of $W$, replace "left zero semigroups" by "left groups". Let $(r, s) \rightarrow f_{r, s}$ be a mapping of $X^{2}$ into $W$. Replace II by the condition $\mathrm{II}^{\prime} f_{s, t}\left(g_{z^{\prime}} \alpha_{s t}\right)=g_{z^{\prime}} \alpha_{t} \alpha_{s} f_{s\left(t z^{\prime}\right)^{\prime}, t z^{\prime}}$, where $g_{z^{\prime}} \in T_{z^{\prime}}$. Add the conditions: I(c) $f_{k, r} \in T_{(k r)^{\prime}} ; \mathrm{I}(\mathrm{d}) f_{s^{\prime}, s} \in E\left(T_{s^{\prime}}\right)$, the set of idempotents of $T_{s^{\prime}} ; \mathrm{I}(\mathrm{e})$ if $s \in X$, there exists $g_{s^{\prime}} \in E\left(T_{s^{\prime}}\right)$ such that $g_{s^{\prime}} \alpha_{s} \in E\left(T_{s^{\prime}}\right)$; III $f_{s . t} f_{s t, z}=f_{t, z} \alpha_{s} f_{s(t z)^{\prime}, t z}$. The multiplication becomes $\left(g_{s^{\prime}, s}\right)\left(h_{t^{\prime}}, t\right)=$ $\left(g_{s^{\prime}}\left(h_{t^{\prime}}, \alpha_{s}\right) f_{s t^{\prime}, t}, s t\right)$. A proof is given in [4]. A semigroup $E$ is a band of maximal left groups if and only if $E$ is a union of groups and $\mathscr{L}$ is a congruence on $E$.

\section{REFERENCES}

1. A. H. Clifford, Semigroups admitting relative inverses, Ann. of Math. (2) 42 (1941), 1037-1049. MR 3, 199. 
2. A. H. Clifford and G. B. Preston, The algebraic theory of semigroups. Vol. I, Math. Surveys, no. 7, Amer. Math. Soc., Providence, R.I., 1961. MR 24 \#A2627.

3. David McLean, Idempotent semigroups, Amer. Math. Monthly 61 (1954), 110-113. MR 15, 681.

4. R. J. Warne, Bands of maximal left groups (to appear).

Department of Mathematics, University of Alabama, Birmingham, Alabama 35233 